\title{
Characterization of a fast piezoelectric varifocal MEMS mirror
}

\author{
Paul Janin, Ralf Bauer, Paul Griffin, Erling Riis, and Deepak Uttamchandani \\ University of Strathclyde, Glasgow, United Kingdom \\ paul.janin@strath.ac.uk
}

\begin{abstract}
We present the characterization of a novel design for a varifocal MEMS mirror with piezoelectric actuation and defocus movement up to $100 \mathrm{kHz}$. The device was simulated using a finite-element method, fabricated using a multi-user silicon-oninsulator process, and its mechanical response to piezoelectric actuation evaluated through laser vibrometry and a dynamic white-light interferometer.
\end{abstract}

Index Terms-optical MEMS, piezoelectric, varifocal mirror, high frequency

\section{INTRODUCTION}

The need for high-frequency beam steering devices for a multitude of scanning applications has brought interest to the development of fast and efficient MEMS scanning mirrors [1]. Such devices are also needed for rapid light addressing of particle arrays, for example in quantum computing applications [2]. Varifocal micromirrors allow further beam control by shifting the focal point of the beam along its propagation axis, potentially enabling 3D steering and addressing. Such devices have been demonstrated using electrostatic [3], electrothermal [4] and piezoelectric [5] actuators, typically for static or low frequency deformations under $20 \mathrm{kHz}$. We present a highfrequency varifocal mirror with piezoelectric actuation capable of both static and resonant operation in the high $\mathrm{kHz}-$ range.

\section{DEVICE DESIGN}

The presented device is a varifocal micromirror, fabricated using MEMSCAP's PiezoMUMPs process with a $10 \mu \mathrm{m}$ thick silicon-on-insulator (SOI) wafer. The device shown in Fig. 1 has a $2.70 \mathrm{~mm}$ diameter, $10 \mu \mathrm{m}$ thick circular silicon disc with a full backside release through a substrate DRIE step. A thin $(500 \mathrm{~nm})$ film of aluminum nitride is deposited in a ring on this circular plate, with an inner diameter of $1.50 \mathrm{~mm}$ and outer diameter of $2.50 \mathrm{~mm}$. This piezoelectric layer is covered with $1 \mu \mathrm{m}$ of aluminium, forming the top electrode of the piezoelectric actuator, with the doped silicon layer forming the bottom electrode. The $1.4 \mathrm{~mm}$ diameter central active mirror area is also coated with aluminium for increased reflectivity.

Actuation of the mirror is controlled by four silicon beams connecting the plate and aluminium electrode to bonding pads. Four pads, one for each beam, are connected to the silicon layer, while two pads connected to the northwest and southeast beams are insulated from the SOI by a silicon oxide layer, and

P.J. was supported by NPL through a NPL Research Studentship and R.B. by the Royal Academy of Engineering under the Engineering for Development Research Fellowship scheme (RF1516 \15\8) control the piezoactuator top electrode. These six control pads have the potential to also allow the actuation of the mirror through electrothermal control. The investigated piezoelectric actuation is achieved by applying a voltage to the aluminium nitride ring.

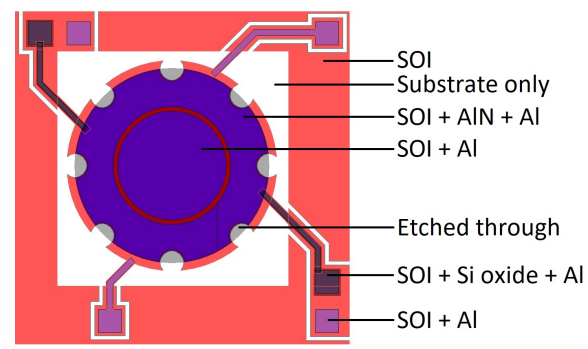

Fig. 1: Layout schematic of the device.

\section{Simulation Results}

The device's frequency response was first simulated using COMSOL Multiphysics. The simulation is taking into account the mechanical structure of the plate, neglecting any fluid damping or heating effects from actuation. The theoretical eigenvalues for the mirror's harmonic response show a first spherical resonance mode shape at $27.3 \mathrm{kHz}$ and a second spherical resonance mode shape at $105.0 \mathrm{kHz}$ (see Fig. 2).
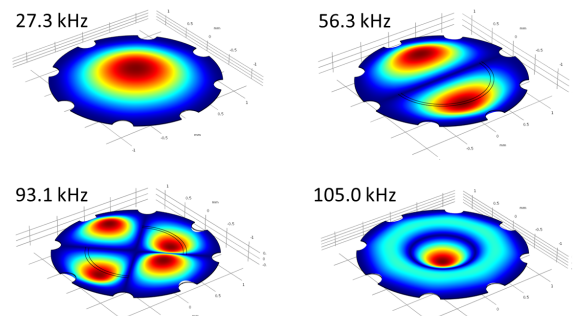

Fig. 2: Simulated mode shapes of the four first eigenfrequencies. first spherical mode at $27.3 \mathrm{kHz}$, coma mode at $56.3 \mathrm{kHz}$, secondary astigmatic mode at $93.1 \mathrm{kHz}$, and secondary spherical mode at 105.0 $\mathrm{kHz}$.

\section{EXPERIMENTAL CHARACTERIZATION}

After fabrication, the properties of the mirror were characterized using laser Doppler vibrometry to measure the velocity response of the mirror at a single point of the plate and white 
light interferometry to examine the static and dynamic mode shapes.

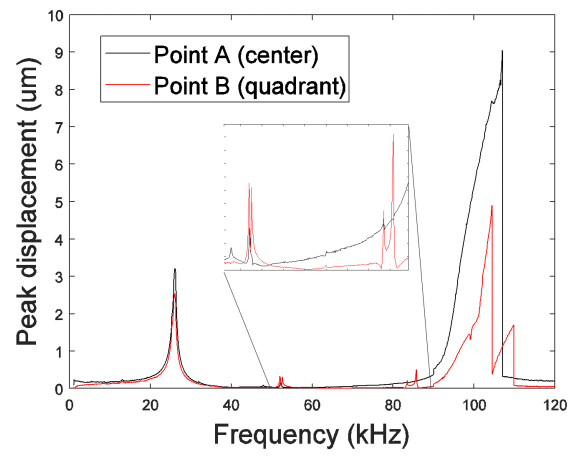

Fig. 3: Velocity response of the varifocal mirror under sinusoidal actuation. Note the hysteretic behaviour around the fourth resonant mode.

The results of the vibrometry measurements, using a Polytec OFV512 vibrometer, are shown in Fig. 3. The mirror was actuated using a $20 \mathrm{~V}_{p p}$ sine-wave centred around $0 \mathrm{~V}$ with 100 $\mathrm{Hz}$ frequency steps. The plot represents the peak displacement measured for a given frequency, with point $\mathrm{A}$ at the centre of the mirror and point $\mathrm{B}$ at the maximum displacement of the first two astigmatic movement mode shapes. The vibrometer measured the velocity of the plate at the corresponding point, from which the displacement amplitude was calculated. This led to the first resonant mode at $26.1 \mathrm{kHz}$ with a displacement amplitude of $3.2 \mu \mathrm{m}$. The movement corresponding to the first four resonant modes can be seen, with the measured resonance frequencies about $10 \%$ below the simulated frequencies for the first three modes. This can be explained by manufacturing imperfections, as well as damping effects not taken in account in the simulations. Furthermore, the degeneracy of the second and third modes can be seen, with the frequencies of each degenerated mode slightly offset seen as two distinct peaks. The fourth resonance mode is measured at $107 \mathrm{kHz}$, with centre displacement of $9.1 \mu \mathrm{m}$.

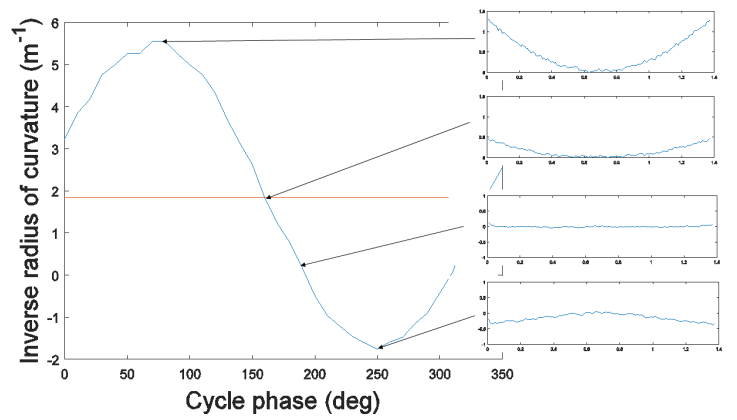

Fig. 4: Inverse radius of curvature of the mirror along an actuation period at $26 \mathrm{kHz}$. The horizontal red line indicates the inverse radius of curvature of the mirror at rest. The insets show the diameter profile of the mirror and are on scale with each other.

The specific mode shape of the resonances were investigated using a Veeco NT1100 dynamic white-light surface profiler.
Fig. 4 shows the radius of curvature variation of the mirror through the resonant movement phase when actuated at the first resonance frequency of $26.1 \mathrm{kHz}$. The radius of curvature shows the central point of the mirror having a sinusoidal displacement centered around the non-actuated curvature of the mirror. The profiles show that the measured displacement is indeed the primary spherical mode (see insets on Fig. 4).

The device response to a DC voltage piezoelectric actuation was measured using the same surface profiler, with the resulting inverse radius of curvature for a voltage range up to $30 \mathrm{~V}$ shown in Fig. 5. The static response of the mirror

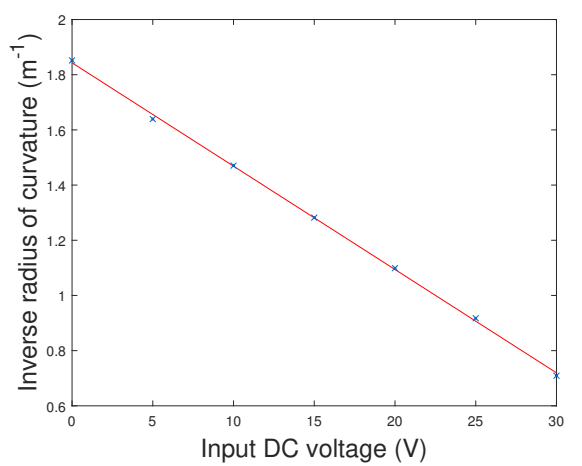

Fig. 5: Inverse radius of curvature of the mirror as a function of input DC voltage.

appears to be linear, with the mirror flattening with increased voltage. While the resonance actuation allows for a fast control over a range of positive and negative Dioptre values, the static curvature control still allows a significant curvature change, with piezoelectric actuation also enabling an inverse response with increase in curvature through an applied voltage.

\section{CONCLusions}

We have presented our work on a piezoelectric actuated varifocal MEMS mirror with resonance actuation in the high $\mathrm{kHz}$ range. The presented mirror with $1.4 \mathrm{~mm}$ active mirror surface has a spherical deformation resonance at $107 \mathrm{kHz}$, allowing fast control of the mirror focal position in a range over $7 \mathrm{D}$ while still having a static focal change response over more than $1 \mathrm{D}$.

\section{REFERENCES}

[1] S. T. Holmstrom, U. Baran, and H. Urey, "Mems laser scanners: a review," Journal of Microelectromechanical Systems, vol. 23, no. 2, pp. 259-275, 2014.

[2] C. Knoernschild, C. Kim, F. P. Lu, and J. Kim, "Multiplexed broadband beam steering system utilizing high speed mems mirrors," Optics express, vol. 17, no. 9, pp. 7233-7244, 2009.

[3] T. Liu and D. L. Dickensheets, "Mems 3-dimensional scanner for handheld confocal microscope," in Optical MEMS and Nanophotonics (OMN), 2017 International Conference on. IEEE, 2017, pp. 1-2.

[4] A. Paterson, R. Bauer, L. Li, W. Lubeigt, and D. Uttamchandani, "Range extension of a bimorph varifocal micromirror through actuation by a peltier element," IEEE Journal of Selected Topics in Quantum Electronics, vol. 21, no. 4, pp. 72-78, 2015.

[5] M. J. Mescher, M. L. Vladimer, and J. J. Bernstein, "A novel high-speed piezoelectric deformable varifocal mirror for optical applications," in $\mathrm{Mi}$ cro Electro Mechanical Systems, 2002. The Fifteenth IEEE International Conference on. IEEE, 2002, pp. 511-515. 